\title{
Reduced Complexity Spatio-Temporal Image-Based Tracking for Maneuvering Targets
}

\author{
Vikram Krishnamurthy and Subhrakanti Dey \\ Department of Electrical and Electronic Engineering, \\ University of Melbourne \\ Victoria 3010, Australia \\ vikram@ee.mu.oz.au and s.dey@ee.mu.oz.au
}

\section{Introduction}

There is significant motivation to develop reduced complexity filtering algorithms (with explicit performance bounds) for tracking maneuvering targets. Maneuvering target estimation is an important problem in target tracking due to the uncertainty in maneuvers of the target. In a hostile environment a target will try to avoid being tracked by maneuvering in such a way so that its motion is difficult to follow. The idea behind image-based and image enhanced tracking $[1,2,3]$ is to use two-dimensional imagery to obtain information about the mode of the target (e.g. orientation information) apart from conventional measurements. Simulation studies in $[1,2,3]$ demonstrate that this modal information can lead to marked improvements in the target tracking performance.

As is widely done $[1,2,3]$, we assume the mode of the target with time is modelled as a finite state Markov chain and the target's trajectory is modelled as a jump Markov linear system. The image sensor processor response to the modal information is blurred to due the range of the target, weather conditions, etc. Finally, the blurred images are processed by an imager which generates a marked Poisson process according to the noisy state of the Markov chain. In summary the image-based target tracking model is a multivariate Poisson process modulated by a single finite state Markov chain, i.e., a Markov Modulated Poisson Process (MMPP).

Estimating the target's mode and coordinates then involves two filtering algorithms: (i) The optimal (MMSE) estimate of the orientation is computed by a MMPP filter (which is essentially a continuoustime Hidden Markov Model filter). (ii) The trajectory of the target (modelled as a jump Markov linear system) given the noisy modal measurements is estimated using an image-based filter. This is a finite dimensional filter (i.e. given by an ordinary differen- tial equation driven by a Poisson observation process) which requires estimates from the MMPP filter [1].

The main contributions of this paper are to present robust reduced complexity temporal and spatial approximations to the above MMPP and image-based filters.

1. Robust Temporal Discretization; We present a robust time discretization of MMPP filter and the optimal image-based filter. In particular, we use a gauge transformation to convert the filtering equations to deterministic differential equations with random coefficients. Such robust transformations have been widely used in stochastic calculus and continuous-time HMM filtering [4]. A major advantage of our approach is that the discretization interval of the resulting robust filters can be much larger than that required by a standard Euler first order discretization of the filter. This leads to substantial computational savings in the numerical implementation of the MMPP and image-based filters. We present explicit upper bounds on the discretization interval for the two filters.

2. Spatial Aggregation: The computation complexity of the robust time discretized MMPP and image based filters is a major issue when the state space is large. This happens for example when there are several modes or a large number of targets each with a finite number of maneuver types. We present a novel algebraic methodology (similarity transformation) for reducing the complexity of both filters. Under the natural assumption that the target's dynamics are similar when the target's modes are similar the underlying Markov chain has a nearly completely decomposable structure [5]. With $\epsilon>0$ denoting a perturbation parameter signifying the weak interactions between dissimilar target modes, our novel similarity transformation decouples the components of the MMPP filter and image-based filters resulting in low complexity filtering algorithms with provable $O(\epsilon)$ accuracy. In order 
to prove the existence of the similarity transformation we demonstrate the existence of solutions to certain asymmetric algebraic Riccati equations. The decoupling transformations are a generalization of the results in [6].

\section{Signal Model}

All processes are defined on the probability space $(\Omega, \mathcal{F}, P)$.

Target Model: Let $\left\{X_{t}, t \geq 0\right\}$ be a continuoustime $S$ state Markov chain defined on the state space $\left\{e_{1}, e_{2}, \ldots, e_{S}\right\}$ where $e_{i} \in \mathbb{R}^{S}$ is the unit vector with 1 in the $i$-th position. (This choice of state space simplifies our subsequent notation). Let the infinitesimal generator or transition rate matrix be denoted by $A$ where $\sum_{j=1}^{S} a_{i j}=0, \forall i \in\{1,2, \ldots, S\}$. Define $P\left(X_{t}=i\right)=p_{t}^{i}, i \in\{1,2, \ldots, S\}$. The probability distribution $p_{t}=\left(p_{t}^{1} p_{t}^{2} \ldots p_{t}^{S}\right)^{\prime}$ satisfies the forward equation $\frac{d p_{t}}{d t}=A^{\prime} p_{t}$ where $/$ denotes the transpose operation. The process $\left\{X_{t}\right\}$ denotes the mode or regime of operation of the target and drives the jump linear system for the target dynamics as:

$$
d s_{t}=c\left(X_{t}\right) s_{t} d t+R d w_{t}
$$

Here $s_{t} \in \mathbb{R}^{L}$ denotes the coordinates of the target and the matrices $c\left(e_{i}\right), i=1, \ldots, S$ are each $L \times L$ matrices. $R$ denotes an arbitrary known matrix. $\mathbf{E}\left\{s_{0}\right\}$ is assumed known and $\left\{w_{t}\right\}$ denotes a Wiener process which is independent of $\left\{X_{t}\right\}$. (If $\mathbf{E}\left\{s_{0}\right\}$ is not known, it can be estimated via an associated stochastic optimization problem - see [2]).

Image Sensor: The image sensor uses twodimensional imagery to obtain orientation information of the target. (For example, [3] considers the profile of a T-62 tank with three different orientations). The output of the image sensor is a $S$-variate Markov modulated Poisson process as outlined below (see $[3,7]$ for excellent expositions): The image sensor is modelled in two steps. First, the appearance of an image frame depends on the mode dependent rates $\lambda^{(i)}$, $i=1, \ldots, S$. Second, the image sensor processor generates an output statement $\rho_{t} \in\left\{e_{1}, \ldots, e_{S}\right\}$. Because of the blurring of the image due to range of the target, weather conditions, etc, the output statement $\rho_{t}$ is not necessarily the same as the true target mode $X_{t}$. This error is modelled probabilistically in terms of the $S \times S$ discernability matrix $D=\left(d_{i j}\right)$, where $d_{i j}=P\left(\rho_{t}=e_{i} \mid X_{t}=e_{j}\right), 1 \leq i, j \leq S$.

As a result the output of the imager is a $S$-variate Markov modulated Poisson process (MMPP) $N_{t}=$ $\left(N_{t}^{(1)} N_{t}^{(2)} \ldots N_{t}^{(S)}\right)^{\prime}$ as follows:

$$
d N_{t}^{(i)}=\left\langle X_{t}, g^{(i)}\right\rangle d t+d m_{t}^{(i)}, i=1,2, \ldots, S .
$$

Here $N_{t}^{(i)}$ denotes the number of events with mark $i$ that occur during the interval $[0, t]$, and $g^{(i)}=$ $\left(g_{1}^{(i)} g_{2}^{(i)} \ldots g_{S}^{(i)}\right)^{\prime}=\lambda^{(i)}\left[\begin{array}{lll}d_{i 1} & \ldots & d_{i S}\end{array}\right]^{\prime}, i=1, \ldots, S$ is the vector of intensities of the $i$-th component of the process $N_{t}$. In (2), $\langle\cdot, \cdot\rangle$ denotes the scalar product in $\mathbb{R}^{S}$ and $m_{t}^{(i)}$ is a $\mathcal{F}_{t}$ Poisson martingale where $\mathcal{F}_{t}$ denotes $\sigma\left(X_{s}, N_{s} ; s \leq t\right)$. Denote the observation history as $\mathcal{N}_{t}^{(i)}=\sigma\left(N_{s}^{(i)}: s \leq t\right)$ and $\mathcal{N}_{t}=\bigvee_{i=1}^{S} \mathcal{N}_{t}^{(i)}$.

The following somewhat technical assumption which essentially states that the jumps of the Markov chain and Poisson process cannot occur at eactly the same time instant is widely assumed [3] and is necessary for deriving the filters presented here. The assumption can be relaxed as in [1], however, the resulting filters then need careful accounting of the co-quadratic variation of $X_{t}$ and the individual processes $N_{t}^{(i)}$.

Assumption 2.1 We assume that $X_{t}, N_{t}^{(1)}, N_{t}^{(2)}, \ldots, N_{t}^{(S)}$ do not have simultaneous jumps, i.e, $\left[X, N^{(i)}\right]_{t}=0, \forall i \in\{1,2, \ldots, S\}$ where $[Y, Z]_{t}$ denotes the optional co-quadratic variation of the process $Y, Z$. Also, assume that $\left[N^{(i)}, N^{(j)}\right]_{t}=0, \forall i, j \in\{1,2, \ldots, S\}, i \neq j$.

\section{Example: Switching Turn Rate Model}

Consider tracking an agile maneuvering target in two-dimensions. Denote the state vector $s=$ $\left[\begin{array}{llll}x & \dot{x} & y & \dot{y}\end{array}\right]^{\prime}$ with $x$ and $y$ denoting the $x, y$ Cartesian coordinates of the horizontal plane.

A target moving with constant speed can be described by the continuous-time stochastic differential equation (1) with

$$
C\left(X_{t}\right)=\left[\begin{array}{cccc}
0 & 1 & 0 & 0 \\
0 & 0 & 0 & -\omega\left(X_{t}\right) \\
0 & 0 & 0 & 1 \\
0 & \omega\left(X_{t}\right) & 0 & 0
\end{array}\right], \quad R=\left[\begin{array}{ll}
0 & 0 \\
1 & 0 \\
0 & 0 \\
0 & 1
\end{array}\right] .
$$

There are two accelerations modeled above, an omnidirectional (white) acceleration described by the vector Wiener process $w_{t}$, and the structured maneuver acceleration represented by the turn rate processes $\omega\left(X_{t}\right)$ which switches between a finite number of possible turn rates.

\section{Robust time discretization of Filters}

\subsection{Preliminaries}

Results regarding the estimation of the state $X_{t}$ given the observation history $\mathcal{N}_{t}$ were derived in [8] and later generalized in [1]. Here, we briefly state the Zakai form (un-normalized filtered density) for computing the optimal mode estimate $\mathbf{E}\left\{X_{t} \mid \mathcal{N}_{t}\right\}$ and optimal target trajectory $\mathbf{E}\left\{s_{t} \mid \mathcal{N}_{t}\right\}$, see [1] for details. 
Define the measure $\bar{P}$ such that

$$
\begin{aligned}
\left.\frac{d P}{d \bar{P}}\right|_{\mathcal{F}_{t}}=\Lambda_{t} & =\prod_{i=1}^{S} \prod_{0 \leq r \leq t}\left\langle g^{(i)}, \dot{X}_{r}\right\rangle\left(\Delta N_{r}^{(i)}\right) \\
& \times \exp \left(-\int_{0}^{t}\left[\left\langle g^{(i)}, X_{r}\right\rangle-1\right] d r\right)
\end{aligned}
$$

Then the following results hold:

(i) $\Lambda_{t}$ is a $\left(\bar{P}, \mathcal{F}_{t}\right)$ martingale. and satisfies $\Lambda_{t}=1+$ $\left.\sum_{i=1}^{S} \int_{0}^{t} \Lambda_{t}\left(\left\langle g^{(i}\right), X_{r}\right\rangle-1\right) d n_{r}^{(i)}[8$, pp.171].

(ii) A straightforward invocation of Girsanov's theorem [8, Theorem T2, pp.166] yields that under $\bar{P}$, each of the $S$ components of $N_{t}$ are independent unit-intensity Poisson processes.

(iii) Let $\bar{E}$ denotes the expectation operator under $\bar{P}$. Define the unnormalized density $q_{t}=\overline{\mathbf{E}}\left\{\Lambda_{t}\left\langle\phi, X_{t}\right\rangle \mid \mathcal{N}_{t}\right\}$ - note $q_{t} \in \mathbb{R}^{N}$ with non-negative elements. If $\phi_{t}$ is a $\mathcal{F}_{t}$ adapted process then an abstract version of Bayes' theorem states $[9, \mathrm{pp} .243]$ that

$$
\mathbf{E}\left\{\left\langle\phi, X_{t}\right\rangle \mid \mathcal{N}_{t}\right\}=\frac{\overline{\mathbf{E}}\left\{\Lambda_{t}\left\langle\phi, X_{t}\right\rangle \mid \mathcal{N}_{t}\right\}}{\overline{\mathbf{E}}\left\{\Lambda_{t} \mid \mathcal{N}_{t}\right\}}=\frac{\left\langle\phi, q_{t}\right\rangle}{\mathbf{1}_{S}^{\prime} q_{t}}
$$

Notation: Define the $S \times S$ diagonal matrix $B^{(i)}, i=$ $1, \ldots, S$ and $(L S) \times(L S)$ matrix $C$, respectively as

$$
\begin{aligned}
B^{(i)}=\operatorname{diag}\left[g^{(i)}\right], \quad C & =\left[\begin{array}{ccc}
C_{11} & \ldots & C_{1 L} \\
\vdots & \ddots & \vdots \\
C_{L 1} & \ldots & C_{L L}
\end{array}\right] \\
\text { where } C_{i j} & =\operatorname{diag}\left[c_{i j}\left(e_{1}\right), \ldots, c_{i j}\left(e_{S}\right)\right]
\end{aligned}
$$

and $c_{i j}\left(e_{l}\right)$ denotes the $i, j$ element of $c\left(e_{l}\right)$ defined in (1).

Also let $n_{t}^{(i)}=N_{t}^{(i)}-t$.

Below we give the Zakai filtering equations for estimating the target mode $X_{t}$ and the target state $s_{t}$. As shown in [1] to estimate the target state $s_{t}$ one first needs to construct the filtered estimate of $s_{t} \otimes x_{t}$ where $\otimes$ denotes the tensor product. Accordingly, define the $L S$ dimensional process

$$
r_{t}=\overline{\mathbf{E}}\left\{\Lambda_{t}\left(s_{t} \otimes X_{t}\right) \mid \mathcal{N}_{t}\right\}
$$

Theorem 1 (Zakai equations for Mode and State)

$$
\begin{aligned}
d q_{t} & =A^{\prime} q_{t} d t+\sum_{i=1}^{S}\left(B^{(i)}-I\right) q_{t} d n_{t}^{(i)} \\
d r_{t} & =C r_{t} d t+\left(I_{L} \otimes A^{\prime}\right) r_{t} d t \\
& =\sum_{i=1}^{S}\left(I_{L} \otimes\left(B^{(i)}-I\right)\right) r_{t} d n_{t}^{(i)} \\
\mathbf{E}\left\{s_{t} \mid \mathcal{N}_{t}\right\} & =\frac{\overline{\mathbf{E}}\left\{\Lambda_{t} s_{T} \mid \mathcal{N}_{t}\right\}}{\overline{\mathbf{E}}\left\{\Lambda_{t} \mid \mathcal{N}_{t}\right\}}=\frac{1}{1^{\prime} q_{t}} \operatorname{diag} \underbrace{\left\{\mathbf{1}_{S}^{\prime}, \ldots, 1_{S}^{\prime}\right.}_{L \text { times }}\} r_{t}
\end{aligned}
$$

The proof of the above theorem appears in $[1$, Sec.5.2]. Note that the second equality in (6) follows because $\overline{\mathbf{E}}\left\{\Lambda_{t} s_{t} \mid \mathcal{N}_{t}\right\}=\overline{\mathbf{E}}\left\{\sum_{i=1}^{S} \Lambda_{t} s_{t} X_{t}(i)\right\}=$ $\operatorname{diag}\left\{\mathbf{1}_{S}^{\prime}, \ldots, \mathbf{1}_{S}^{\prime}\right\} r_{t}$ (where the diagonal matrix is of dimension $L \times L S$ ) and $\overline{\mathbf{E}}\left\{\Lambda_{t}\right\}=\overline{\mathbf{E}}\left\{\sum_{i=1}^{S} \Lambda_{t} X_{t}(i)\right\}=$ $\mathbf{1}_{S}^{\prime} q_{t}$.

\subsection{Robust Filters and Discretization}

The aim of this section is to transform the stochastic differential equations (4), (5) into linear ordinary differential equations (ODEs) with random coefficients. This permits the use of standard time discretization techniques for ODEs and computationally efficient numerical implementation.

For $i=1, \ldots, S$ define

$\Gamma_{t}^{(i)}=\left(B^{(i)}\right)^{N_{t}^{(i)}} \exp \left(-\left(B^{(i)}-I\right) t\right), \quad \Gamma_{t}=\prod_{i=1}^{S} \Gamma_{t}^{(i)}$

Define the robust variables $\bar{q}_{t}$ and $\bar{r}_{t}$ as

$$
\bar{q}_{t}=\Gamma_{t}^{-1} q_{t}, \quad \bar{r}_{t}=\left(I_{L} \otimes \Gamma_{t}\right)^{-1} r_{t}
$$

Theorem $2 \bar{q}_{t}$ and $\bar{r}_{t}$ satisfy the linear ODEs

$$
\begin{array}{ll}
\frac{d \bar{q}_{t}}{d t}=\Gamma_{t}^{-1} A^{\prime} \Gamma_{t} \bar{q}_{t}, \quad \bar{q}_{0}=q_{0} \\
\frac{d \bar{r}_{t}}{d t}=\left(I_{L} \otimes \Gamma_{t}\right)^{-1}\left(I_{L} \otimes A^{\prime}+C\right)\left(I_{L} \otimes \Gamma_{t}\right) \bar{r}_{t}, & \bar{r}_{0}=r_{0}
\end{array}
$$

\section{Discretization Interval for Robust Filters}

We now consider time discretization of the above robust equations (9) and (10). Consider a regular time partition $0=t_{0}<t_{1}<\ldots<t_{n-1}<t_{n}<\ldots$ with constant time step $\Delta=t_{n}-t_{n-1}$. Define the discretetime observation probability diagonal matrix

$$
B_{t_{n+1}}=\operatorname{diag}\left[b_{t_{n+1}}(1), \ldots, b_{t_{n+1}}(S)\right] \triangleq \Gamma_{t_{n+1}} \Gamma_{t_{n}}^{-1}
$$

Note that $B_{t_{n+1}}$ is pre-computable since

$$
B_{t_{n+1}}=\prod_{i=1}^{S}\left[\left(B^{(i)}\right)^{\left(N_{t_{k+1}}^{(i)}-N_{t_{k}}^{(i)}\right)} \exp \left(-\left(B^{(i)}-I\right) \Delta\right)\right]
$$

and for sufficiently fine discretization $N_{t_{k+1}}^{(i)}-N_{t_{k}}^{(i)}$ is either 0 or 1 :

A first order (Euler) explicit discretization of (9) yields:

$$
\bar{q}_{t_{n+1}}=\bar{q}_{t_{n}}+\Delta \Gamma_{t_{n}}^{-1} A^{\prime} \Gamma_{t_{n}} \bar{q}_{t_{n}}
$$

Multiplying both sides by $\Gamma_{t_{n+1}}$ yields the discretetime robust mode filter

$$
q_{t_{n+1}}=B_{t_{n+1}}\left(I+A^{\prime} \Delta\right) q_{t_{n}} .
$$


Similarly, a first order explicit discretization of (10) followed by multiplying both sides by $\left(I_{L} \otimes \Gamma_{t}\right)$ yields the discrete-time robust image filter

$$
r_{t_{n+1}}=\left(I_{L} \otimes B_{t_{n+1}}\right)\left[I_{L} \otimes\left(I_{S}+A^{\prime} \Delta\right)+C \Delta\right] r_{t_{n}} .
$$

Note that for all $n, q_{t_{n+1}}$ should be a $S$ dimensional vector with non-negative elements. Similarly, consider $r_{t}$ in (10). If $\bar{r}_{0}>0$ (element wise) then from (10), $\bar{r}_{t} \geq 0$ for all $t$. This is easily seen since the RHS of (10) is 0 when $r_{t}=0$, meaning that 0 is an absorbing point and hence the components of $r_{t}$ can never go negative. Our aim is to determine an upper bound for the size of the time discretization step $\Delta$ in (12) and (13) to ensure non-negativity of the discretized processes (12) and (13).

Theorem 3 To ensure non-negativity of the elements of the discretized processes $q_{t_{n}}$ in (12) requires the sampling period $\Delta$ to satisfy

$$
\Delta \leq \frac{1}{\max _{j \in\{1, \ldots, S\}}\left|a_{j j}\right|}
$$

To ensure non-negativity of the elements of the discretized processes $r_{t_{n}}$ in (13) requires $\Delta$ to satisfy

$$
\Delta \leq \frac{1}{\max _{m}\left|a_{i i}+c_{m m}\right|} \text { where } i=m \bmod S
$$

\section{Discretization Interval for Non-robust Filters}

Here we consider the Euler discretization of the $\mathrm{Za}$ kai (non-robust) equations (4) and (5). In the interval $t_{n}<t \leq t_{n+1}$

$q_{t_{n+1}}=q_{t_{n}}+\int_{t_{n}}^{t_{n+1}} A^{\prime} q_{s} d s+\int_{t_{n}}^{t_{n+1}} \sum_{i=1}^{S}\left(B^{(i)}-I\right) q_{s} d n_{s}^{(i)}$

First order discretization and normalization with $\hat{q}_{t_{n}}=$ $q_{t_{n}} /\left(\mathbf{1}^{\prime} q_{t_{n}}\right)$ yields

$$
\begin{aligned}
& \hat{q}_{t_{n+1}}(j)=\hat{q}_{t_{n}}(j)+\sum_{j=1}^{S} \Delta a_{i j} \hat{q}_{t_{n}}(i) \\
& +\sum_{i=1}^{S}\left(g_{j}^{(i)}-1\right) \hat{q}_{t_{n}}(j)\left(\Delta N_{t}^{(i)}-\Delta\right), \quad t_{n}<t \leq t_{n+1}
\end{aligned}
$$

Again considering the case $\hat{q}_{t_{n}}=e_{j}$, it follows that

$$
\Delta \leq \frac{1+\sum_{i=1}^{S}\left(g_{j}^{(i)}-1\right) \Delta N_{t}^{(i)}}{\sum_{i=1}^{S}\left(g_{j}^{(i)}-1\right)+\left|a_{j j}\right|}
$$

providing $\sum_{j=1}^{S} g_{j}^{(i)}-1>a_{j j}$.
Consider now the Zakai equation of the image filter: (5). Using similar steps to above it follows that

$$
\Delta \leq \frac{1+\sum_{i=1}^{S}\left(g_{j}^{(i)}-1\right) \Delta N_{t}^{(i)}}{\sum_{i=1}^{S}\left(g_{j}^{(i)}-1\right)-\left(a_{j j}+c_{m m}\right)}, \quad j=m \bmod S
$$

providing that $\sum_{i=1}^{S}\left(g_{j}^{(i)}-1\right)>\left(a_{j j}+c_{m m}\right)$ for $j \epsilon$ $\{1, \ldots, S\}$ and $m \in\{1, \ldots, L S\}$.

\section{Comparison}

Consider the case when $\Delta N_{t}^{(i)}=0$, i.e., no event has occurred at time $t$. Then comparing (14) with (17) or (15) with (18) shows that $\Delta$ for the robust filters are independent of the Poisson rates. Thus the non-robust filters require substantially smaller sampling intervals if the Poisson rates $g_{j}^{(i)}$ are large. As a result the robust filters are substantially more efficient from a computational point of view.

\section{Reduced-complexity state and modal estimation}

Having demostrated the computational efficiency of the robust filters, in this section we describe spatial aggregation methods to further decrease the computational complexity of the mode and image filters. Throughout we will work with the robust filters (12) and (13). We will exploit a transformation method proposed in [10] [6] followed by the decoupling transformation used in [6] to obtain approximate reduced-complexity computations for the target trajectory (state) and modal (state of the underlying Markov chain) estimates. It is shown that for the case where the underlying Markov chain is nearly completely decomposable (with a sufficiently small weak coupling parameter $\epsilon$ ), one can obtain $O(\epsilon)$ approximations to the MMPP and image based filters when the Poisson arrival rates and the target dynamics are the same for all the constitutent states within a given mode but are different from one mode to another. Of course, one could prove similar results for a (polynomial in $\epsilon^{2}$ ) perturbation in these arrival rates and target dynamics provided $\epsilon$ is sufficiently small.

For the purpose of this section, we then impose the following nearly decomposable structure on the underlying Markov chain: $A=\bar{A}+\epsilon Q$ where $\bar{A}$ has a block diagonal structure

$$
\bar{A}=\left[\begin{array}{ccccc}
\bar{A}_{11} & 0 & . & \cdot & 0 \\
0 & \bar{A}_{22} & 0 & \cdot & \cdot \\
0 & 0 \cdot & . & \cdot & \cdot \\
\cdots & . & \cdot & \cdot & \cdot \\
0 & \cdot & 0 & \cdot & \bar{A}_{M M}
\end{array}\right]
$$


where $\bar{A}_{i i} \in \mathbb{R}^{s_{i} \times s_{i}}, \forall i, \sum_{i} s_{i}=S, \epsilon>0$ is a small perturbation parameter, and $Q \in \mathbb{R}^{S \times S}$. $\bar{A}_{i i}, \forall i$ are also infinitesimal generators and $\bar{B}$ has zero row-sums. Denote the state partitions as $\mathcal{S}_{1}=$ $\left(e_{1}, e_{2}, \ldots, e_{s_{1}}\right), \mathcal{S}_{2}=\left(e_{s_{1}+1}, e_{s_{1}+2}, \ldots, e_{s_{1}+s_{2}}\right)$ and so on. We assume that $A$ and the individual infinitesimal generators $A_{i i}, \forall i$ represent irreducible Markov chains. We also make another crucial assumption (as in [6]):

Assumption 4.1 The intensities of the Poisson arrival process and the target dynamics are only different from one mode to another but are identical for all constituent states within a given mode. In other words, $g_{l}=\bar{g}_{m}, \forall X_{t}=e_{l} \in \mathcal{S}_{m}$. Similarly, $c_{i j}\left(e_{l}\right)=\bar{c}_{i j}\left(e_{m}\right)$ $\forall X_{t}=e_{l} \in \mathcal{S}_{m}, i, j \in\{1,2, \ldots, L\}, m \in\{1,2, \ldots, M\}$.

Notice that Assumption 4.1 also guarantees that the elements of $B_{t_{n+1}}$ are identical within each mode. Now, rewrite (12), (13) by using $\alpha_{t_{n+1}}=q_{t_{n+1}}^{\prime}, \beta_{t_{n+1}}=$ $r_{t_{n+1}}^{\prime} ;$ as

$$
\begin{gathered}
\alpha_{t_{n+1}}=\alpha_{t_{n}}\left(I_{S}+A \Delta\right) B_{t_{n+1}} \\
\beta_{t_{n+1}}=\beta_{t_{n}}\left[I_{L} \otimes\left(I_{S}+A \Delta\right)+C^{\prime} \Delta\right]\left(I_{L} \otimes B_{t_{n+1}}\right)
\end{gathered}
$$

In the following discussion, we denote the corresponding normailized measures for $\alpha_{t_{n+1}}, \beta_{t_{n+1}}$ as $\hat{\alpha}_{t_{n+1}}, \hat{\beta}_{t_{n+1}}$. In the next two subsections, we describe how one can obtain reduced-complexity computations for $\hat{\alpha}_{t_{n+1}}$ and $\hat{\beta}_{t_{n+1}}$. These computations are of $O\left(M^{2}\right)$ instead of $O\left(S^{2}\right)$ as required by the exact computations. Since typically, $M \ll S$, this implies a large savings in computations. These computations result in an $O(\epsilon)$ approximation to $\hat{\alpha}_{t_{n}}$ and $O\left(\epsilon^{2}\right)$ approximation to $\hat{\beta}_{t_{n}}$.

\section{Reduced-complexity modal estimation for the MMPP filter}

Denote $\left[\zeta_{n} \eta_{n}\right]=\alpha_{t_{n}}\left[W_{1} W_{2}\right]$ where $W_{1} \in \mathbb{R}^{S \times M}$ is given by

$$
W_{1}=\left[\begin{array}{cccc}
1_{s_{1}} & 0 & 0 & 0 \\
0 & 1_{s_{2}} & \cdot & \cdot \\
\cdot & 0 & \cdot & 0 \\
0 & \cdot & \cdot & 1_{s_{M}}
\end{array}\right]
$$

Similarly, $W_{2} \in \mathbb{R}^{S \times(S-M)}$ is a block diagonal matrix with the $i$-th diagonal block $W_{2}^{(i)} \in \mathbb{R}^{s_{i} \times s_{i}-1}$ is given by

$$
W_{2}^{(i)}=\left[\begin{array}{c}
0 \ldots 0 \\
I_{s_{i}-1}
\end{array}\right]
$$

Note that for these choices $\left[W_{1} W_{2}\right]$ is nonsingular and has an inverse $\left[\begin{array}{l}V_{1} \\ V_{2}\end{array}\right]$ where $V_{1}, V_{2}$ are also simple matrices independent of the system parameters. Notice an obvious fact that $\zeta_{n} \in \mathbb{R}^{M}$ is the aggregate version of the unnormalized measure $\alpha_{t_{n}}$. Also, similar relationships can be established between the normalized measures $\hat{\zeta}_{n}, \hat{\eta}_{n}$ with $\hat{\alpha}_{t_{n}}$. It is easy to show that $\hat{\alpha}_{t_{n}}=\hat{\zeta}_{n} V_{1}+\hat{\eta}_{n} V_{2}$.

Also, define

$\tilde{A}_{1}^{n}=V_{1}(I+\bar{A} \Delta) B_{t_{n+1}} W_{1}, \tilde{Q}_{1}^{n}=V_{1}(Q \Delta) B_{t_{n+1}} W_{1}$

$\tilde{A}_{2}^{n}=V_{1}(I+\bar{A} \Delta) B_{t_{n+1}} W_{2}, \tilde{Q}_{2}^{n}=V_{1}(Q \Delta) B_{t_{n+1}} W_{2}$

$\tilde{C}_{1}^{n}=V_{2}(I+\bar{A} \Delta) B_{t_{n+1}} W_{1}, \tilde{D}_{1}^{n}=V_{2}(Q \Delta) B_{t_{n+1}} W_{1}$

$\tilde{C}_{2}^{n}=V_{2}(I+\bar{A} \Delta) B_{t_{n+1}} W_{2}, \tilde{D}_{2}^{n}=V_{2}(Q \Delta) B_{t_{n+1}} W_{2}$

In fact, under Assumption 4.1, one can show that $C_{1}^{n}=0, \forall n$. Using these definitions and notations, one can then write the recursion (19) in terms of $\left[\zeta_{n} \eta_{n}\right]$ and then decouple $\eta_{n}$ from $\zeta_{n}$ by using the following decoupling transformation:

$$
\left[\begin{array}{ll}
\bar{\zeta}_{n} & \bar{\eta}_{n}
\end{array}\right]=\left[\begin{array}{ll}
\zeta_{n} & \eta_{n}
\end{array}\right]\left[\begin{array}{cc}
I_{M} & J_{n} \\
0 & I_{S-M}
\end{array}\right]
$$

where (see [6] for more details)

$\left[\tilde{A}_{1}^{n}+\epsilon\left(\tilde{Q}_{1}^{n}-J_{n} \tilde{D}_{1}^{n}\right)\right] J_{n+1}=J_{n}\left(\tilde{C}_{2}^{n}+\epsilon \tilde{D}_{2}^{n}\right)-\left(\tilde{A}_{2}^{n}+\epsilon \tilde{Q}_{2}^{n}\right)$

where for a sufficiently small $\epsilon$, one can show that $\left\{J_{n}\right\}$ is a sequence of uniformly bounded matrices (see [6] for a proof). Thus one can expand $J_{n}$ in terms of a power series of $\epsilon$, i.e, $J_{n}=J_{n}(0)+\epsilon J_{n}(1)+\ldots$. One can also show that $J_{n}(0) \rightarrow J(0)$ as $n \rightarrow \infty$ where $J(0)=V_{1} \bar{A} W_{2}\left(V_{2} \bar{A} W_{2}\right)^{-1}$. Finally, one can write $O\left(\epsilon^{2}\right)$ approximations for $\hat{\zeta}_{n}, \hat{\eta}_{n}$ (hence $\hat{\alpha}_{n}$ ) as a result of this decoupling.

Note that the approximations are written with $\mathrm{a}^{\text {- }}$ sign, that is, an approximation for normalized measures like $\hat{\zeta}_{n}$ is written as $\tilde{\zeta}_{n}$, whereas the corresponding approximation for an unnormalized measure like $\zeta_{n}$ is written as $\tilde{\zeta}_{n}^{u}$ where the superscript $u$ stands for "unnormalized". We now present our main result regarding the reduced-complexity $O(\epsilon)$ computations for the robust discretized Zakai filter $\alpha_{t_{n+1}}$ :

Theorem 4 Consider the Markov modulated Poisson process described by (2) where the modulating Markov chain has a nearly completely decomposable structure $A=\bar{A}+\epsilon Q$ and the transition probability matrices $A, \bar{A}_{i i}$ are irreducible $\forall i$. Suppose Assumption 4.1 holds. Then, for a sufficiently small $\epsilon$, there exists a large enough but finite $n_{0}$ such that for $n \geq n_{0}$, an $O(\epsilon)$ approximation for the robust discretized approximate Zakai filter (19), denoted by $\tilde{\alpha}_{t_{n}}$ can be described by the following recursions:

$$
\begin{aligned}
& \tilde{\zeta}_{n+1}^{u}=\tilde{\zeta}_{n}\left[\tilde{A}_{1}^{n}+\epsilon\left(\tilde{Q}_{1}^{n}-J(0) \tilde{D}_{1}^{n}\right)\right], \tilde{\zeta}_{n_{0}-1}=\hat{\zeta}_{n_{0}-1} \\
& \tilde{\zeta}_{n+1}=\frac{1}{\left[\tilde{\zeta}_{n+1}^{u} 1_{M}\right]} \tilde{\zeta}_{n+1}^{u}
\end{aligned}
$$




$$
\begin{gathered}
\tilde{\eta}_{n}=-\tilde{\zeta}_{n} J(0), \quad \tilde{\eta}_{n_{0}-1}=\hat{\eta}_{n_{0}-1} \\
\tilde{\alpha}_{t_{n}}=\tilde{\zeta}_{n} V_{1}+\tilde{\eta}_{n} V_{2}, \tilde{\alpha}_{t_{n_{0}-1}}=\hat{\alpha}_{t_{n_{0}-1}}
\end{gathered}
$$

Proof See [6].

Remark 1 Notice that in the above approximations, one can actually obtain an $O\left(\epsilon^{2}\right)$ approximation to $\zeta_{n}$ (or $\hat{\zeta}_{n}$ ) but an $O(\epsilon)$ approximation to $\hat{\eta}_{n}$ and hence an $O(\epsilon)$ approximation to $\hat{\alpha}_{t_{n}}$. One can obtain an $O\left(\epsilon^{2}\right)$ approximation to $\hat{\eta}_{n}$ and hence $\hat{\alpha}_{t_{n}}$ at a slightly higher computational cost. For the above recursions, notice that the computational cost is $O\left(M^{2}\right)$ per time instant (as opposed to $O\left(S^{2}\right)$ per time instant for exact computations).

\section{Reduced-complexity estimation for the target trajectory}

For the purpose of this section define

$$
\left[\xi_{n}^{(1)} \chi_{n}^{(1)} \ldots \xi_{n}^{(L)} \chi_{n}^{(L)}\right]=\beta_{t_{n}}\left(I_{L} \otimes\left[W_{1} W_{2}\right]\right)
$$

One can then use a generalized decoupling transmformation to define (for $i=1,2, \ldots, L$ )

$$
\left[\bar{\xi}_{n}^{(i)} \bar{\chi}_{n}^{(i)}\right]=\left[\xi_{n}^{(i)} \chi_{n}^{(i)}\right]\left(\begin{array}{cc}
I_{S} & \bar{J}_{n}^{i} \\
0 & I_{S-M}
\end{array}\right)
$$

Rewriting the recursion (20) in terms of the decoupled variables, and after denoting $G_{n+1}^{i i}=$ $\Delta C_{i i} B_{t_{n+1}}, G_{n+1}^{i j}=\Delta C_{j i} B_{t_{n+1}}, \bar{J}_{n}^{i}, i=1,2, \ldots, L$ satisfy certain collection of nonlinear recursive and simultaneous (pairwise) linear equations (under Assumption 4.1).

Under Assumption 4.1, one can show that the first order approximations to $\bar{J}_{n}^{i}, i=1,2, \ldots, L$ (i.e, when $\epsilon=0$ ) (that satisfy these equations) denoted by $\bar{J}_{n}^{i}(0) \rightarrow J(0), \forall i$ as $n \rightarrow \infty$ (this result is similar to that in the previous subsection and the case presented in [6]). Hence, for a sufficiently small $\epsilon$, using analyticity arguments, $\left\{J_{n}^{i}\right\}, \forall i$ can be shown to be sequences of uniformly bounded sequences of matrices which can be approximated by $J(0)$ for a sufficiently large $n$. Note that in [6], we provide explicit inequalities to obtain ranges for $\epsilon$ to prove a similar uniform boundedness result. In this paper, we refrain from obtaining such results for two reasons: (1) these inequalities are quite complicated and (2) solving them to obtain the desired range of $\epsilon$ can be quite cumbersome as well. Some simulation results are presented to illustrate that one can indeed use this approximation in practice to obtain reduced-complexity computations for the image-based filter recursions (20). In fact, one can obtain $O\left(\epsilon^{2}\right)$ recursions to the normalized measures $\hat{\xi}_{n}^{(1)}, \hat{\xi}_{n}^{(2)}, \ldots, \hat{\xi}_{n}^{(L)}$ and hence the normalized measure $\hat{\beta}_{t_{n}}$ for a sufficiently large $n$. This result is summarized in the following theorem. The proof is analogous to that of Theorem 4 and is omitted.

Theorem 5 Consider the target model given by (1) and the Markov modulated Poisson process given by (2) with an underlying Markov chain that is nearly completely decomposable with $A=\bar{A}+\epsilon Q$ where $A$, $\bar{A}_{i i}, \forall i=1,2, \ldots, M$ satisfy the irreducibility conditions. Suppose also that Assumption 4.1 holds. Then there exists a sufficiently large integer $n_{1}>0$ such that for $n \geq n_{1}$, one can obtain $O\left(\epsilon^{2}\right)$ approximations to the normalized measures $\hat{\xi}_{n}^{(i)}$ and $\hat{\beta}_{t_{n}}$ (and hence to the robust discretized target trajectory estimator $\hat{r}_{t_{n}}$ ) using the following approximate computations denoted by $\tilde{\xi}_{n}^{(i)}, \tilde{\beta}_{n}$ which are given by (for $n \geq n_{1}$ ) (with the unnormalized estimates denoted with $a^{-}$) $(i, j=1,2, \ldots, L)$

$$
\begin{aligned}
\tilde{\xi}_{n+1}^{(i)}= & \tilde{\xi}_{n}^{(i)}\left(V_{1}\left[\left(I_{S}+\bar{A} \Delta\right) B_{t_{n+1}}+G_{n+1}^{i i}\right] W_{1}\right. \\
& \left.\quad+\epsilon\left(Q_{n}^{1}-J(0) D_{n}^{1}\right)\right)+\sum_{j \neq i} \tilde{\xi}_{n}^{j}\left(V_{1} G_{n+1}^{j i} W_{1}\right) \\
\tilde{\xi}_{n+1}^{(i)}= & \tilde{\xi}_{n+1}^{(i)} /\left(\tilde{\zeta}_{n+1}^{u} 1_{M}\right) \\
\tilde{\beta}_{n+1}= & {\left[\tilde{\xi}_{n+1}^{(1)} \ldots \tilde{\xi}_{n+1}^{(L)}\right]\left(I_{L} \otimes 1_{M}\right) }
\end{aligned}
$$

Remark 2 Note that the order of approximation for $\tilde{\beta}_{n}$ is $O\left(\epsilon^{2}\right)$ as opposed to an $O(\epsilon)$ approximation to the MMPP filter as described in Theorem 4.

\section{Numerical Examples}

In this section, we present numerical examples to illustrate the performance of our reduced-complexity mode estimation and image enhanced target trajectory estimation schemes.

These simulations are carried out with the following choice of parameters for the signal model described in Section 2. The continuous-time system equations (refer to the actual equation) are approximated by their Euler discretizations. We adopted an underlying nearly completely decomposable Markov chain with a generator matrix $A=\bar{A}+\epsilon \bar{Q}$ where

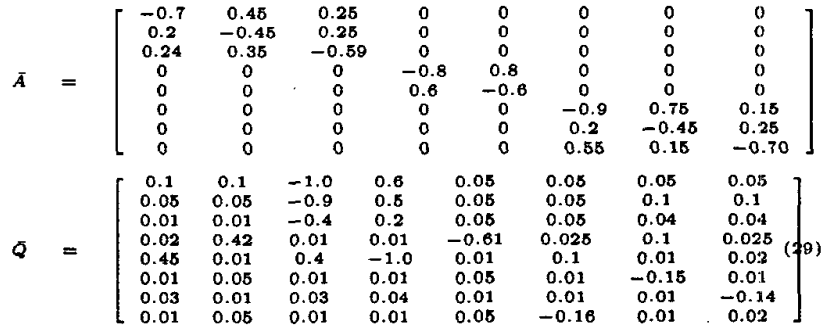

Clearly, for this example, $S=8, M=3$. The Markov modulated Poisson arrival rates are given by

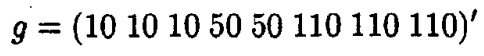


where we have chosen $g(1)=g(2)=g(3), g(4)=$ $g(5)$ and $g(6)=g(7)=g(8)$. Similarly, the target trajectory is approximated by an Euler discretization of (refer to the equation) by

$$
s(k)=s(k-1)+\Delta C\left(X_{k}\right) s(k-1)+R w_{k}
$$

where $s(k) \triangleq(x \dot{x} y \dot{y}) \in \mathbb{R}^{4}$ which is also known as the switching turn rate model of a maneuvering target in two dimensions $x, y$. The sampling time interval $\Delta$ is taken to be 0.001 second, $w_{k}$ is a zero mean white Gaussian sequence with covariance $Q=\sigma^{2} I_{2}$. The matrix $R$ is given by

$$
R=\left[\begin{array}{cc}
0.5 \Delta^{2} & 0 \\
\Delta & 0 \\
0 & 0.5 \Delta^{2} \\
0 & \Delta
\end{array}\right]
$$

Here $w_{k}$ models an omni-directional acceleration process whereas $C(1)=C(2)=C(3)=\left[\begin{array}{llll}0 & 1 & 0 & 0 \\ 0 & 0 & 0 & 0 \\ 0 & 0 & 0 & 1 \\ 0 & 0 & 0 & 0\end{array}\right]$ describe a constant velocity process for the modes $1,2,3$. Similarly $C(4)=C(5)$ and $C(6)=C(7)=C(8)$ describe two different turn rate processes with

$$
C(4)=\left[\begin{array}{cccc}
0 & 1 & 0 & 0 \\
0 & 0 & 0 & -\omega_{1} \\
0 & 0 & 0 & 1 \\
0 & \omega_{1} & 0 & 0
\end{array}\right]
$$

and

$$
C(6)=\left[\begin{array}{cccc}
0 & 1 & 0 & 0 \\
0 & 0 & 0 & -\omega_{2} \\
0 & 0 & 0 & 1 \\
0 & \omega_{2} & 0 & 0
\end{array}\right]
$$

with $\omega_{1}=\pi / 3 \mathrm{radians} / \mathrm{second}$ and $\omega_{2}=-\pi / 3 \mathrm{ra-}$ dians/second. The simulations are carried out with a 10000 point Markov chain (i.e, over a period of 10 seconds). The results are averaged over 10 simulation trials.

Table 1 shows the average approximation error between the exact aggregate modal filter $\xi_{k}$ and the reduced-order approximate aggregate modal filter $\tilde{\xi}_{k}$ measured as $\frac{1}{T} \sum_{k=1}^{T} \sqrt{\left\|\xi_{k}-\tilde{\xi}_{k}\right\|^{2}}$ against various values of $\epsilon$ between 0.001 and 0.15 . Similarly, Table 2 shows the average relative approximation error in image enhanced trajectory estimation between the exact filter and the reduced-order filter.

Figures 1, 2 show snapshots of the true trajectory (solid line), the estimated trajectory (the "dashdotted" line) according to the exact image-enhanced filter and the estimated trajectory (the "dashed" line)

\begin{tabular}{|c|c|}
\hline$\epsilon$ & Average error in aggregate modal filtering \\
\hline 0.001 & $1.0594 \times 10^{-6}$ \\
\hline 0.005 & $1.042 \times 10^{-5}$ \\
\hline 0.01 & $4.204 \times 10^{-5}$ \\
\hline 0.05 & $1.554 \times 10^{-4}$ \\
\hline 0.1 & $6.6468 \times 10^{-4}$ \\
\hline 0.15 & $1.3 \times 10^{-3}$ \\
\hline
\end{tabular}

Table 1: Average approximation error in lowcomplexity aggregate modal filtering

\begin{tabular}{|c|c|}
\hline$\epsilon$ & Average error in image enhanced filtering \\
\hline 0.001 & $2.3929 \times 10^{-10}$ \\
\hline 0.005 & $4.361 \times 10^{-9}$ \\
\hline 0.01 & $1.097 \times 10^{-7}$ \\
\hline 0.05 & $4.387 \times 10^{-7}$ \\
\hline 0.1 & $2.8 \times 10^{-6}$ \\
\hline 0.15 & $3.2872 \times 10^{-5}$ \\
\hline
\end{tabular}

Table 2: Average relative error in low-complexity image enhanced filtering

according to the reduced-order image enhanced filter, for $\sigma^{2}=0.01$ and $\sigma^{2}=0.1$, respectively (with $\epsilon=0.1$ ). It is clear that the filters perform quite well with the exact filter and the reduced-order filter being almost indistinguishable. Note that although here we assume the "block"-structured measurement dynamics ( $g$ and $C$ matrices being dependent only on the super-states of the Markov chain), small perturbations of such dynamics will result in similar performances of the reducedorder filters.

\section{References}

[1] V. Krishnamurthy and R. Elliott, "Filters for estimating Markov modulated poisson processes and image based tracking," Automatica, vol. 33, pp. 821-833, May 1997.

[2] F. Dufour and P. Bertrand, "An image based filter for discrete-time Markovian jump linear systems," Automatica, vol. 32, pp. 241-247, 1996.

[3] D. D. Sworder and R. G. Hutchins, "Imageenhanced tracking," IEEE Trans. Aerospace and Electronic Systems, vol. 25, pp. 701-709, Sept. 1989.

[4] A. Bensoussan, Stochastic Control of Partially Observable Systems. Cambridge, UK: Cambridge University Press, 1992.

[5] C. Meyer, "Stochastic complementation, uncoupling Markov chains, and the theory of nearly re- 


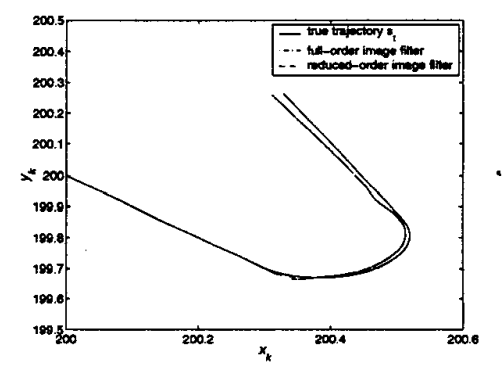

Figure 1: Low-complexity image enhanced target trajectory estimation, $\sigma^{2}=0.01$

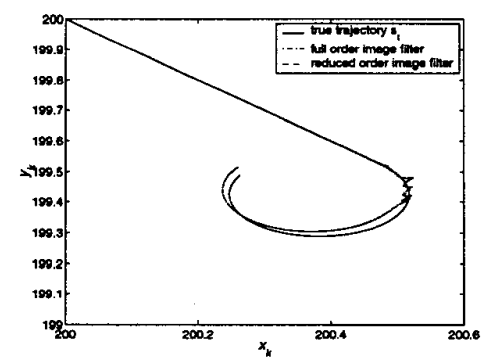

Figure 2: Low-complexity image enhanced target trajectory estimation, $\sigma^{2}=0.1$

ducible systems," SIAM Review, vol. 31, pp. 240$272,1989$.

[6] S. Dey, "Reduced-complexity filtering for partially observed nearly completely decomposable Markov chains", IEEE Trans. Signal Proc., vol. 48, pp. 3334-3344, December 2000.

[7] C. Yang, Y. Bar-Shalom, and C.-F. Lin, "Discrete-time point process filter for mode estimation," IEEE Trans. Auto. Control, vol. 37, pp. 1812-1816, Nov. 1992.

[8] P. Bremaud, Point Processes and Queues. New York: Springer-Verlag, 1981.

[9] E. Wong and B. Hajek, Stochastic Processes in Engineering Systems. Berlin: Springer-Verlag, second ed., 1985.

[10] R. Aldhaheri and H. Khalil, "Aggregation of the policy iteration method for nearly completely decomposable Markov chains," IEEE Transactions on Automatic Control, vol. AC-36, no. 2, pp. 178187, 1991. 